DRAFT VERSION MARCH 25, 2022

Preprint typeset using LATEX style emulateapj v. 5/2/11

\title{
TIDAL DISRUPTIONS IN CIRCUMBINARY DISCS (I): STAR FORMATION, DYNAMICS, AND BINARY EVOLUTION
}

\author{
Pau Amaro-Seoane ${ }^{1}$, Patrick Brem ${ }^{1} \&$ Jorge CuAdra $^{2,3}$ \\ (Dated: March 25, 2022) \\ Draft version March 25, 2022
}

\begin{abstract}
In our current interpretation of the hierarchical structure of the universe it is well established that galaxies collide and merge with each other during their lifetime. If massive black holes (MBHs) reside in galactic centres, we expect them to form binaries in galactic nuclei surrounded by a circumbinary disc. If cooling is efficient enough, the gas in the disc will clump and trigger stellar formation in situ. In this first paper we address the evolution of the binary under the influence of the newly formed stars, which form individually and also clustered. We use SPH techniques to evolve the gas in the circumbinary disc and to study the phase of star formation. When the amount of gas in the disc is negligible, we further evolve the system with a high-accurate direct-summation $\mathrm{N}$-body code to follow the evolution of the stars, the innermost binary and tidal disruption events (TDEs). For this, we modify the direct $N$-body code to (i) include treatment of TDEs and to (ii) include "gas cloud particles" that mimic the gas, so that the stellar clusters do not disolve when we follow their infall on to the MBHs. We find that the amount of stars disrupted by either infalling stellar clusters or individual stars is as large as $10^{-4} / \mathrm{yr}$ per binary, higher than expected for typical galaxies.
\end{abstract}

\section{INTRODUCTION}

Super-massive black hole (MBHs) binaries are expected to form after major galaxy mergers. The main driving mechanism for the MBHs to sink to the centre is dynamical friction, where they will form a binary and start to shrink the semimajor axis on their way to the final merger. Slingshot of stars from the surrounding stellar environment help the binary to further decay by exchanging energy and angular momentum, down to distances of about $1 \mathrm{pc}$ (Begelman et al. 1980). However, if the amount of stars to interact with is depleted, there is a risk of stalling, so that the MBHs would not coalesce within a Hubble time. This is the so-called "last-parsec problem" (see Merritt \& Milosavljević 2005 for a review on the whole process and references therein).

Key factors to surmount this last "snag" in the evolution are, among others, the fact that (i) in the case of binaries with a total mass of $\leq 10^{7} M_{\odot}$, slingshot ejections suffice to guarantee coalescence within a Hubble time (Milosavljević \& Merritt 2003); (ii) the role of gas may be crucial in the evolution of the binary, starting at larger scales. It might well be that in a merger of gas-rich galaxies, if MBHs are present, they will coalesce soon after the galaxies merge, in some $10^{7} \mathrm{Myr}$, if the gas is distributed spherically. If, on the other hand, the gas is forming a nuclear disc, the galaxies need only to have $1 \%$ of their total mass in gas for this to happen. (Escala et al. 2004. 2005). Cuadra et al. (2009) found that such gas discs could indeed commonly help in the merger of SMBHs with masses in the range of our study, whilst this mechanism fails for masses larger than $\sim 10^{7} M_{\odot}$; (iii) following with stellar dynamics, resonant relaxation creates a steady state current of stars which can be as large as ten times the non-coherent twobody relaxation (Hopman \& Alexander 2006). This is a potential source of new stars populating the depleted loss-cone;

\footnotetext{
${ }^{1}$ Max Planck Institut für Gravitationsphysik (Albert-Einstein-Institut), D-14476 Potsdam, Germany

${ }^{2}$ Departamento de Astronomía y Astrofísica, Facultad de Física, Pontificia Universidad Católica de Chile, 782-0436 Santiago, Chile

${ }^{3}$ Max-Planck Institut für Astrophysik, Karl-Schwarzschild-Str. 1, D85741 Garching bei München, Germany
}

(iv) the work of Berczik et al. (2006) shows that considering a non-spherically symmetric system the final parsec problem is largely solved (v) massive perturbers, such as giant molecular clouds or intermediate-mass black holes, can accelerate relaxation by orders of magnitude compared to two-body stellar relaxation, so that many new stars are supplied to interact with (Perets \& Alexander 2008); (vi) it has been observed that young, compact star clusters such as the Arches and Quintuplet systems reside near the Galactic centre. If these star clusters have masses larger than $10^{5} M_{\odot}$, they can make their way down to the Galactic centre even if they start from a distance as large as $60 \mathrm{pc}$ within a few million years (McMillan \& Portegies Zwart 2003). The tidal stripping of these young stars could eventually provide the binary system with a new set of some $\approx 10^{5}$ stars; (vii) if intermediate-mass black holes (IMBHs), with masses ranging between $10^{2-4} M$. exist in the centre of clusters, it has been predicted that within the innermost central $10 \mathrm{pc}$, we can expect to have some 50 IMBHs of masses $10^{3} M_{\odot}$, and still some of them at scales of a few milliparsecs (Portegies Zwart et al. 2006). The interaction of one of these IMBHs with the binary of SMBHs would obviously accelerate the process of shrinkage.

The studies just cited provide a number of mechanisms to make the binary shrink. We expect then that a typical binary will be able to reach sub-pc separations, especially in the case of relatively low-mass MBHs in gas-rich environments. In this study, we concentrate on such a case (see section 2.1 for details), which is expected when the parent galaxies are gasrich and large amounts of gas fall to the centre of the new system, together with the MBHs. At that location, the black holes get bound to each other, thus forming a binary, and are surrounded by a massive, parsec-scale gaseous disc (e.g., Escala et al.|2005; Mayer et al. 2007, Dotti et al. 2007).

Such gaseous discs are similar to proto-stellar discs: due to their high density compared to the central object tidal force, the discs will be locally unstable to self-gravity, meaning that perturbations in their density field will grow. However, if the gas is unable to cool efficiently, then the gas will not be able to contract and form clumps, and the density perturbations will 
be sheared apart, creating a quasi-steady spiral pattern. Remarkably, the spiral pattern transports the angular momentum outward, making the disc behave as an accretion disc. On the contrary, if the gas is able to cool quickly enough, then the density perturbations grow and form clumps, which shrink and further accrete gas, breaking up the gaseous disc completely and turning it into stars - the so-called fragmentation (e.g., Gammie 2001, Rice et al. 2005; Nayakshin et al. 2007; Lodato 2007; Alexander et al. 2008; Paardekooper 2012).

In either cooling regime, the situation where at the centre of the disc the central object is a binary will lead to a non-trivial interaction between them. On previous studies we have focused on the inefficient-cooling regime, showing that torques between the gas and the binary will shrink the orbit of the latter, while the angular momentum is driven out through the disc (Cuadra et al. 2009, Roedig et al. 2011, 2012). In this paper we present the first numerical study of the fast cooling regime in which the disc fragments into stars, and follow the dynamical evolution of the binary-stars system. We carry out our study in two stages (see also Khan et al. 2012): first we model the fragmentation of the disc using smoothed particle hydrodynamics, and then we switch to our direct-summation $N$-body models to both follow the long-term evolution of the system and to study the occurence of TDEs.

The reason for this two-step approach is that we first need to model the gas hydrodynamics in order to follow the fragmentation process of the gas, including the formation of stars and their growth via mergers and accretion of gas. In principle, one could wait for the gas to disperse or be accreted, and simply continue the same integration to follow the dynamical evolution of the stars for long time-scales. However, the SPH code we are using is not designed to follow the collisional Nbody dynamics of the system, therefore, it is necessary to use a different code that allow us to model the system of MBHs and stars in a meaningful way.

\section{FIRST STAGE IN THE EVOLUTION: GASEOUS DISC AND STAR} FORMATION IN SITU

\subsection{Two MBHs and a circumbinary disc}

Following Cuadra et al. (2009), we concentrate on a binary with the following initial parameters: total mass $M_{\mathrm{bbh}}=$ $M_{1}+M_{2}$, where $M_{1}$ and $M_{2}$ are the masses of the individual MBHs, and mass ratio $M_{1} / M_{2}=3$, in a circular (Newtonian) orbit of separation $a$. The binary is surrounded by a corotating gaseous disc with an initial mass $M_{\mathrm{d}}=0.2 M_{\mathrm{bbh}}$ and radial range $2 a-5 a$. The gas is modelled as an ideal gas with $\gamma=5 / 3$, and radiative cooling is mimicked with a cooling time defined as $t_{\text {cool }}(r)=\beta / \Omega(r)$, where $\beta$ is a free parameter that fixes the cooling rate, $\Omega(r)=\sqrt{G M_{\mathrm{bbh}} / r^{3}}$ is the orbital frequency around the binary, and $r$ is the distance from the binary centre of mass. Since we are interested in the fragmentation regime, in this paper we consider fast cooling rates, $\beta \leq 5$. The choice of a disc that fragments is realistic for selfgravitating discs that cool thermally, above a certain surface density threshold. Levin (2007) showed that, for the masses and distances we are interested in here, that threshold lies in the $10-100 \mathrm{~g} / \mathrm{cm}^{2}$ range.

This model for the system dynamics is scale-free, meaning that it can be scaled up or down to different masses and lenghts. However, in order to introduce star formation and also to estimate the rate of tidal disruption events (TDEs), we need to choose physical units. With that aim we set the total mass of the binary as $M_{\mathrm{bbh}}=3.5 \cdot 10^{6} \mathrm{M}_{\odot}$ and we choose $a=0.04 \mathrm{pc}$. This would be a typical mass for binary black holes in the range that could be detected by a LISA-like experiment (Amaro-Seoane et al.2012ab). The chosen separation corresponds roughly to the value where we would expect binaries to spend the longest of their evolution in a simple model that considers binary shrinking due to stellar scattering from a spherical cusp (Milosavljević \& Merritt 2003) and torques from a non-fragmenting disc (see Cuadra et al. 2009 their eq. 12). ${ }^{4}$

While several studies Amaro-Seoane \& Freitag 2006, Amaro-Seoane et al. 2009, 2010; Preto et al. 2011; Khan et al. 2011, 2012) have shown that stellar dynamical processes pump up the eccentricity of a binary $\mathrm{MBH}$, in this case we are assuming the binary has reached the inner parsec in a gas-rich environment. In such a case, the dynamical friction of the gas on the MBHs drives them to form a circular binary (e.g., Dotti et al. (2007)). Thus we choose a circular orbit for the initial configuration.

\subsection{Implementation and treatment of the disc fragmentation}

To follow the process of circumbinary disc fragmentation, we use a modified version of the smoothed particle hydrodynamics (SPH) code (GADGET, Springel et al. 2001; Springel 2005), combining the numerical methods of Nayakshin et al. (2007) and Cuadra et al. (2009). Here we only briefly describe the methods, and refer the interested reader to those papers for more details. We model the gaseous disc as an ensemble of initially $\approx 2 \times 10^{6}$ particles of $\approx 0.35 \mathrm{M}_{\odot}$ each. The code calculates the gravitational and hydrodynamical interaction between gas particles, plus the gravitational interaction between all particles, including the MBHs as well as the "proto-stars" and "stars" that form during the simulation (see below). We use a softening of $0.001 a$ for the gas particles and of $0.01 a$ for the proto-stars. The MBHs do not use softening, but a sink radius within which gas particles are accreted. This radius had a value of $0.3 a$.

As initial conditions, we take the initially-circular system modelled by Cuadra et al. (2009), at a time $T \approx 500 \Omega_{0}^{-1}$. In this way we skip the transient initial evolution caused by the homogeneous initial conditions described in their work, and start from a steady-state configuration in which the circumbinary disc has developed spiral arms. Notice, however, that their simulations used $\beta=10$, avoiding fragmentation. In our new simulations we set the value of $\beta$ to either $1,2,3$, or 5. As a result, the disc now forms clumps, which grow in a runaway fashion. Treating this this with a pure SPH model is not feasible, as the growing densities require ever shorter time-steps. To circumvent this problem, we introduce sink particles to model the proto-stars that we expect would form in these large density regions.

Proto-star particles are created when the gas density reaches 30 times the Roche tidal limit, $M_{\mathrm{bbh}} /\left(2 \pi r^{3}\right)$. How many stars will form out of a gas density peak is a very complex question, whose solution is well outside the possibilities of our study. In our model we deal with this issue in an individual particle basis, i.e., each gas particle is turned into one proto-star particle of the same mass. However, the newly formed proto-star particles can merge with each other, thus forming higher mass stars. The merger criterion is simply that their distance is smaller than $2 f_{\mathrm{m}} R_{\mathrm{p}}$, where $R_{\mathrm{p}}$ is the size

\footnotetext{
${ }^{4}$ Notice that the choice of $a=0.04 \mathrm{pc}$ is below the classical $\sim 1 \mathrm{pc}$ separation of the "final parsec problem", but for the range of masses considered in this work we deem it not a problem, as we summarized in the introduction.
} 
of the proto-stars, which we typically take as $10^{15} \mathrm{~cm}$, and $f_{\mathrm{m}}$ is a free parameter with fiducial value of unity that mimics the effect of gravitational focusing. The size parameter corresponds to $\sim H / 10$ (where $H$ is the disc scale-height), which is roughly the thickness of the gas arms we observe in the simulations. Thus, in a two-step process we are in principle allowing all the dense gas within the same overdensity to form one proto-star. However, we only allow the proto-stars to merge with each other as long as their masses do not exceed $30 \mathrm{M}_{\odot}$. Once they reach this mass we turn the proto-star particle into an actual star particle. The motivation for this threshold is twofold: numerically, we form an actual star out of $\gtrsim 100$ gas particles; physically, we avoid the rapid formation of extremely massive stars. Stars can merge with proto-stars, but not among each other.

Stars and proto-stars also grow by accreting their surrounding gas. We use an Eddington-limited Bondi-Hoyle prescription to calculate their accretion rate, and then pick up at random enough particles from the (proto-)star neighbours that are merged with the sink particle (Springel et al. 2005). To calculate the Bondi-Hoyle and Eddington accretion rates, we use the mass of the (proto) star, and a radius that is either the main sequence value corresponding to that mass (eq. 11 in Nayakshin et al. 2007) for the star particles, or the fixed value $R_{\mathrm{p}}$ for the proto-stars. This difference results in a much faster growth for proto-stars than for stars.

The black holes also accrete the few gas particles that get too close to them. This procedure is done mostly to avoid the short time-steps that would be required to follow those gas particle orbits. Accretion on to the black holes is modelled simply with a sink radius - all gas particles entering the region around $0.3 a$ of either black hole are taken away from the simulation, with their mass and momentum being added to the corresponding MBH (Cuadra et al. 2006).

We have ran 6 different SPH simulations. Four of them use the fiducial values mentioned above, but differ on the strength of the cooling. We refer to these runs as beta1, beta2, beta3a and beta5. Additionally, since we tend to form many very massive stars, we explore the effect of decreasing the numerical size of the proto-stars, hindering their growth. For $\beta=3$ then we run two additional simulations, beta 3 b and beta $3 \mathrm{c}$, in both of which we use a smaller size for the protostars of $1436.8 R_{\odot}$ instead of the fiducial value of $14,368 R_{\odot}$. Run beta $3 \mathrm{c}$ has however a larger gravitational focusing factor of $f_{\mathrm{m}}=10$ instead of the fiducial $f_{\mathrm{m}}=1$. For both extra simulations then there is a more severe (Eddington) limit on the accretion rate for the proto-stars than in the fiducial beta $3 \mathrm{a}$, while simulation bet $\mathrm{a} 3 \mathrm{~b}$ has additionally a smaller likelihood of proto-stellar mergers.

These choices in the conditions for gas cooling and for transforming gas particles into "stars" arguably capture a sufficiently broad number of potential fragmentation scenarios so as to envisage our analysis representative of a self-gravitating disc, within the limitations of the rather expensive numerical experiments.

\section{FRAGMENTING DISCS}

We run the SPH simulations of circumbinary discs for several hundred binary dynamical times. Due to the gas selfgravity, clumps grow in the disc. Given the short cooling times, these clumps contract, achieving the disc fragmentation. In most simulations, after only $\approx 200 \Omega_{0}^{-1}$, the vast ma- jority ( $\gtrsim 90 \%$ ) of the gas is turned into stars, as expected. The system then reaches a quasi-steady state in which stars very slowly accrete the tenous left-over gas (see Nayakshin et al. 2007). The gas morphology at that stage for the different simulations is shown in Fig. 1 .

The fragmentation rate is set by the cooling time of the disc, thus discs with lower values of $\beta$ will evolve faster. We can see this in figure 2, which shows the mass in stars as a function of time for all the simulations. The fourth column in Table 1 shows the number of stars formed in each simulation. Considering only the variation of $\beta$, it is clear that shorter cooling times result in larger amounts of stars, as expected (Nayakshin et al. 2007). As the total stellar mass is approximately constant, the typical stellar masses will be lower for shorter cooling times.

It is interesting to note that the star formation process is not uniform. Instead, it happens preferentially in a few localised, relatively large regions, whose sizes are set by the spiral-arm overdensities. Even though we allow proto-stars to merge when they form close together, our numerical recipe avoids the formation of very large stars, which forces the formation of "stellar clusters" (see the left panel of Fig. 5) 5. Some of these clusters feel a strong torque from the spiral arm and are driven towards the centre of the system, where the tidal force of the binary disperses them. This stellar distribution affects the long-term dynamics of the system and has interesting consequences for the production of tidal disruption events (\$4.1).

In our tests with $\beta=3$ and different stellar growth recipes we first notice that runs bet $a 3 a$ and bet $a 3 c$ are practically identical, and that run beta3b has the same curve of stellar mass growth. From this we conclude that in our simulations accretion is not important and that stellar growth is driven by mergers of sink particles. 6 We also notice that the number of stars formed is about an order of magnitude higher in beta3b, which has 10 times smaller proto-stars than the fiducial run, and that the effect of having smaller proto-stars in the simulation is similar to having a shorter cooling time.

To continue our study of the evolution of the MBHs and circumbinary disc system, we will take the masses, positions, and velocities of all sink particles and use them as input in direct-summation $N$-body simulations. For simplicity, we take the snapshot at time $T=300 \Omega_{0}^{-1}$ for all configurations, except for beta 5. Since in that run the evolution is slower, we use the snapshot at $T=1000 \Omega_{0}^{-1}$, by which time $90 \%$ of the gas has turned into stars.

\section{THE ROLE OF STARS IN THE SHRINKING OF THE BINARY}

To analyse the dynamical evolution of the MBH binary embedded in the stellar system product of the stellar formation we use a direct-summation code, NBODY6. This is a very ex-

5 For a movie of this simulation, visit the URL http://members.aei.mpg.de/amaro-seoane/ fragmenting-discs

The encoding of the movie is the free OGG Theora format and should stream automatically with a gecko-based browser (such as mozilla or firefox) or with chromium or opera. Otherwise please see e.g. http: //en.wikipedia.org/wiki/Wikipedia:Media_help_(Ogg) for an explanation on how to play it.

6 This is actually not surprising, as stars grow by mergers in roughly the dynamical time inside an overdensity, $t_{\text {dyn }} \sim(G \rho)^{-1 / 2}$, which corresponds to about hundred years for the density values required for the introduction of sink particles. On the other hand, the Bondi accretion rate for a solar mass sink, even for those very high densities, is only $\dot{M}_{\text {Bondi }} \sim 10^{-5} M_{\odot} \mathrm{yr}^{-1}$ in our models, so the time required to accrete $a$ single $\mathrm{SPH}$ particle turns out to be $t_{\text {acc }} \sim 3 \times 10^{4} \mathrm{yr}$ 

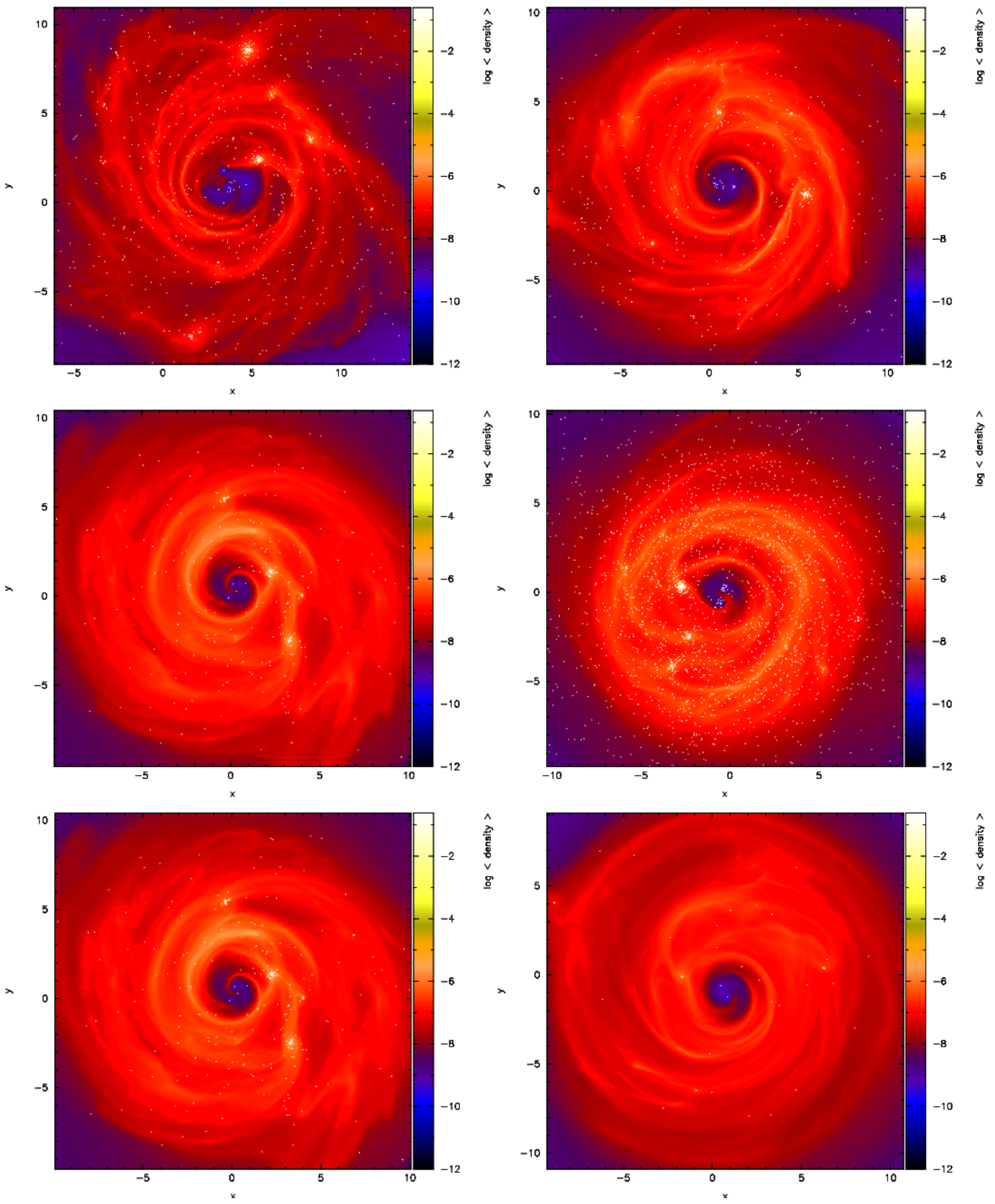

FIG. 1.- Gas density projected in the X-Y plane perpendicular to the angular momentum vector of the system for beta1, beta2, beta3a, beta3b, beta $3 \mathrm{c}$ and beta5, from the left to the right and from the top to the bottom. White dots represent the "sink" particles, i.e. the MBHs and the stars formed during the simulations. All snapshots are at $T=300 \Omega_{0}^{-1}$ but for the last one, which was integrated up to $T=1000 \Omega_{0}^{-1}$, because in that run cooling is quite slow and the number of stars is still very low at earlier times (see Fig.2 2. Note that there is virtually no difference betwen beta3a and beta3c. The figures were made with SPLASH (Price 2007). 


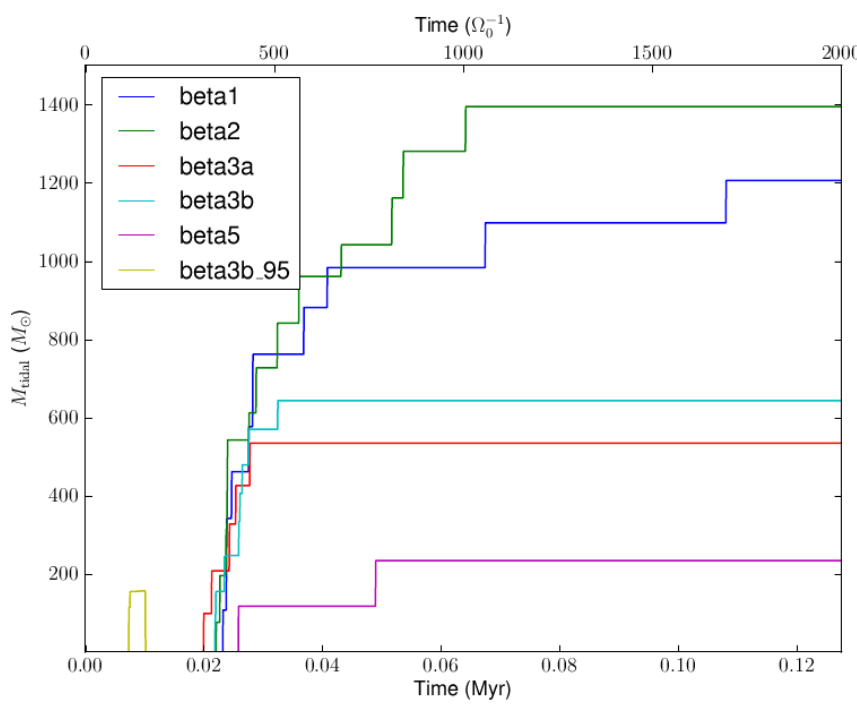

FIG. 2.- Accumulated stellar mass formed in the disc in $M_{\odot}$ for our fiducial case of a binary of $3.5 \cdot 10^{6} M_{\odot}$. All simulations but for $\beta=5$, which needs a bit longer, reach relatively fast the maximum of stellar mass and saturate with values below $10^{6} \mathrm{M}_{\odot}$.

pensive method because we integrate all gravitational forces for all formed stars at every time step, without making any a priori assumptions about the system. This code belongs to the family of dynamical codes for particle systems with relaxation processes of Sverre Aarseth. The code uses the improved Hermite integration scheme as described in (Aarseth 1999. 2003). Since these approaches integrate Newton's equations directly, all Newtonian gravitational effects are included naturally. More crucial for this subject is that it also incorporates both the $K S$ regularisation and the chain regularisation, so that when stars are tightly bound or their separation becomes too small during a hyperbolic encounter, the system is regularised (Kustaanheimo \& Stiefel 1965). The advantages of this code as compared to the leap frog integrator of GADGET for our particular problem are obvious, namely the high accuracy in the energy conservation, since we are interested in the correct evolution of the inner binary of MBHs as well as in potential TDEs. For this aim, as we describe later, we modified the standard version of NBODY6.

For each simulation, the initial masses, coordinates and velocities for the stars and MBHs are taken from the GADGET data at the times shown in table 1 At that moment, the gas mass - stellar mass ratio is very low (see table 1], column $\left.M_{\text {gas }} / M_{\star}\right)$. The gravitational effect of gas is almost negligible and we do not include it in the simulations. Despite our limit to the growth of "proto-star particles" in the SPH simulations (see section 3), some "star particles" did manage to achieve very large masses. We deem those unphysical, so in the initial conditions for our $\mathrm{N}$-body runs we replace stars with masses $m$ above $120 M_{\odot}$ with a cluster following a Plummer distribution (Plummer 1911) consisting of equal mass stars with total mass $m$ and radius

$$
R=\left(\frac{m}{3 M_{\mathrm{bbh}}}\right)^{1 / 3} r
$$

with $r$ the distance to the centre-of-mass of the binary. The last equation corresponds to the Roche lobe of the massive star with respect to the MBH binary with mass $M_{\mathrm{bbh}}$.

In our $N$-body simulations, table 1 , we exclude stars which are at a distance $r>100 a$, where $a$ is the semi-major axis of

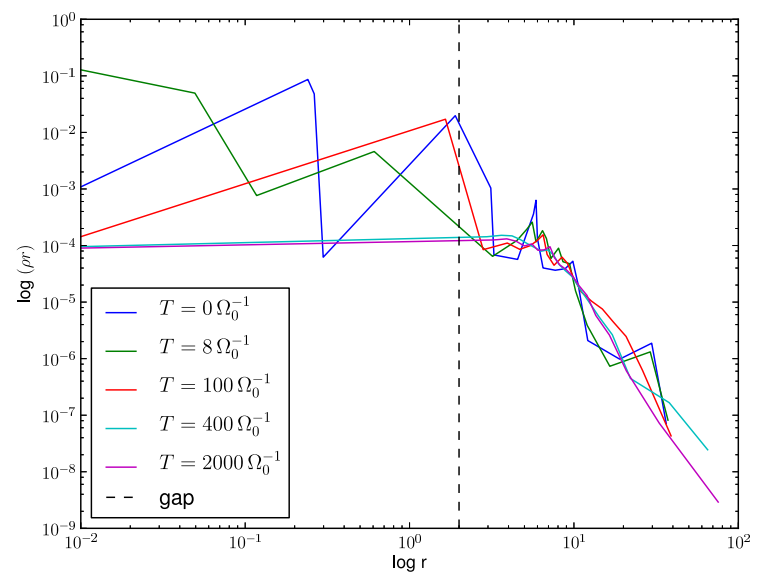

FIG. 3.- Evolution of the density profile in one $N$-body simulation beta1 at different times in the evolution. The dashed line corresponds approximately to the position of the inner gap in the SPH simulation.

\begin{tabular}{|l|l|l|l|l|l|} 
Model & SPH time & $M_{\text {gas }} / M_{\star}$ & $N_{\text {SPH }}$ & $N_{\text {NB }}$ & $N_{\text {split }}$ \\
\hline beta1 & 300 & $3 \%$ & 2536 & 1895 & 4469 \\
\hline beta2 & 300 & $7 \%$ & 1429 & 1141 & 2768 \\
\hline beta3a & 300 & $9 \%$ & 699 & 585 & 1924 \\
\hline beta3b & 300 & $9 \%$ & 5487 & 4486 & 5193 \\
\hline beta5 & 1000 & $10 \%$ & 167 & 144 & 1146 \\
\hline \hline beta3b95 & 95 & - & 5540 & 5540 & 5540 \\
\hline
\end{tabular}

TABLE 1

INITIAL DATA FOR THE NBODY6 RUNS. NOTICE THAT WE DO NOT INTEGRATE RUN beta3c USING THE $N$-BODY TECHNIQUE, BECAUSE IT TURNED OUT TO BE IDENTICAL TO beta3a. SPH TIME IS THE MOMENT AT WHICH WE STOP THE GADGET SIMULATION, IN UNITS OF $\Omega_{0}^{-1}$, $M_{\text {gas }} / M_{\star}$ IS THE RATIO BETWEEN GAS AND STELLAR MASS AT THAT MOMENT, $N_{\text {SPH IS THE NUMBER OF STARS THAT HAVE BEEN FORMED AT }}$ THAT MOMENT IN THE GADGET SIMULATION, $N_{\mathrm{NB}}$ IS THE NUMBER OF STARS WITHIN A DISTANCE $r<100 a$ FROM THE CENTRE OF MASS OF THE BINARY AND $N_{\text {split }}$ IS THE NUMBER THAT WE GET AFTER SPLITTING ALL VERY MASSIVE STARS INTO SUB-CLUSTERS, AS EXPLAINED IN SECTION4 THE REASON WHY THE LAST MODEL HAS MORE STARS THAN beta $3 \mathrm{~b}$ AT $T=300$ IS BECAUSE IT CORRESPONDS TO A PREVIOUS MOMENT IN THE EVOLUTION AND, AS WE EXPLAINED ABOVE, PROTOSTARS ARE ALLOWED TO MERGE WITH EACH OTHER.

THIS LAST CASE IS A SPECIAL ONE, AND WE RAN A DEDICATED SIMULATION FOR IT. SEE SECTION 4.1 ALSO, WE NOTE THAT WHILE THE GAS WAS ORIGINALLY DISTRIBUTED IN A RATHER NARROW RADIAL RANGE $(2 a-5 a)$, WE END UP WITH STARS EVEN AT DISTANCES $>100 a$. THIS IS DUE TO $N$-BODY SCATTERING, AS MANY STAR PARTICLES ARE FORMED IN RELATIVELY CROWDED REGIONS OF THE DISC.

the MBH binary. We assume those stars would have only a negligible effect on the binary evolution. They correspond to about a quarter of all stars in each simulation. As shown in figure 3. this cut in the cluster did not affect its global structure, its density profile remains roughly constant at large radii. The figure also shows that the region inside a few times the binary semi-major axis gets depleted quickly by sling-shot interactions, as expected.

In figure 4 we see the evolution of $a$ and $e$ for all cases, integrated with NBODY6 with the results of the SPH simulations as input parameter. After some 10,000 orbits the binaries reach a stagnation point from which the decay becomes much slower. The decay rates $(1 / a)(\Delta a / \Delta t)$ averaged over the time period from $0.5 \mathrm{Myr}$ to $3 \mathrm{Myr}$ are: betal : $7.2 \times 10^{-9} \mathrm{yr}^{-1}$, beta2 : $4.0 \times 10^{-9} \mathrm{yr}^{-1}$, beta3a : $8.0 \times 10^{-9} \mathrm{yr}^{-1}$, and beta5 : $4.0 \times 10^{-9} \mathrm{yr}^{-1}$ (although for this case we start at 
$1000 \Omega_{0}^{-1}$, which means actually from 0.56 to $3.06 \mathrm{Myr}$ ). In the first $0.1 \mathrm{Myr}$ of the evolution, the significant drop in semi-major axis corresponds to decay rates of $6.7 \times 10^{-7} \mathrm{yr}^{-1}$ for beta1, $5.4 \times 10^{-7} \mathrm{yr}^{-1}$ for beta2, $5.4 \times 10^{-7} \mathrm{yr}^{-1}$ for beta $3 \mathrm{a}$ and $3.2 \times 10^{-7} \mathrm{yr}^{-1}$ for beta 5 .

The early dynamical evolution (first few hundred $\Omega_{0}^{-1}$ ) is dominated by close encounters between the MBH binary and stars on radial orbits (i.e. in the loss cone of the binary). This is naturally accompanied by a high rate of tidal disruptions (see figure 7) and a strong change in orbital binding energy of the binary. In the following long-term evolution, the loss cone has been depleted and the binary is subject to the secular effects of the disk as a non-spherical background potential. The effect of this type of mass distribution is a slow exchange of orbital energy but a rather efficient exchange of angular momentum (Merritt \& Vasiliev 2010), which is consistent with the significant increase in eccentricity that we observe in this phase compared to the very slow decay rates in the semi-major axis.

\subsection{An infalling cluster of young stars}

In the SPH simulations modelling disc fragmentation we see large amounts of stars falling to the immediate vicinity of the MBH binary. In particular, in simulation beta $3 \mathrm{~b}$ we observe an infalling cluster ${ }^{7}$ at $T=95 \Omega_{0}^{-1}$ (see figure 5 .

Since this is quite interesting from the stellar dynamics point of view, we run a dedicated simulation for this particular situation with the direct-summation code. Nonetheless, at this early stage in the evolution of the disc, there is a significant mass in gas which has not yet transformed into stars. If we ran the simulation without taking into account the gas, the small stellar clusters would dissolve, as their potential wells would be abruptly much shallower and the stars could not be held together. We therefore have to include a prescription in the $N$-body simulations for the role of the gas, since including the gas particles directly is well outside the scope of our work.

In this dedicated $N$-body simulation we model each dense region that contains a non-negligible amount of mass as one particle with a big softening length. For this, we define a sphere at every region of interest. We then look at the SPH gas particle distribution and group together all particles within this region, compute their total mass, center-of-mass position and velocity and create one "cloud particle" with these properties (see Fig. 6). In the subsequent $N$-body simulation these particles are integrated separately, which required a modification in the code. In all gravitational interactions, the gravitational potential of the cloud particle seen by a regular star is then computed as

$$
\Phi_{\mathrm{c}}=-\frac{G M_{\mathrm{c}}}{r_{\mathrm{c}}+\epsilon},
$$

where $M_{\mathrm{c}}$ and $r_{\mathrm{c}}$ are the mass and distance to the cloud particle and $\epsilon$ denotes the softening length, taken to be of the order of the size of the corresponding sub-cluster. Although the concept of cloud particles is already implemented in the standard version of NBODY6, we modified it to integrate the cloud particles taking into account the gravitational potential of the other clouds, stars and $\mathrm{MBHs}$ in order to follow correctly the orbits around the central binary of MBHs.

7 As in the former footnote about the movie, from $T=95$ onwards in the simulation.

\begin{tabular}{|l|c|} 
Simulation & TDEs $\left(\mathrm{yr}^{-1}\right)$ \\
\hline \hline beta1 & $1.1 \cdot 10^{-4}$ \\
beta2 & $1.4 \cdot 10^{-4}$ \\
beta3a & $6 \cdot 10^{-5}$ \\
beta3b & $9 \cdot 10^{-5}$ \\
beta5 & $2 \cdot 10^{-5}$ \\
\hline
\end{tabular}

TABLE 2

TIDAL EVENT RATES FOR THE SIMULATIONS OF TABLE 1

The effect is that the particles in the sub-clusters now feel an additional gravitational force corresponding to the cloud and thus stay within their respective group for a longer time, which allows us to study their infall and to analyse TDEs. However, after one close encounter of a gas cloud with one of the MBHs, the cloud would suffer a stripping from the cluster and now float around as an unphysically big agglomeration of mass. This means that we can get only a meaningful result for the very first encounter of each sub-cluster with the binary. In this respect, when estimating the TDEs for the infalling cluster, we will be giving a lower limit, since we cannot simulate realistically further interactions of the cluster with the $\mathrm{MBH}$ binary. In the right panel of figure 6 we show the distribution of stars in the $\mathrm{X}-\mathrm{Y}$ plane after the first interaction.

\section{TIDAL DISRUPTION EVENTS}

During the direct-summation $N$-body runs any star entering the tidal radius $R_{\mathrm{T}}$ of one of the MBHs is considered to be tidally disrupted and its mass is added to the mass of the hole. For a solar-type star, this radius is (see e.g. Amaro-Seoane 2012, for a derivation and examples)

$$
R_{\mathrm{T}}=R_{\star}\left(\frac{M_{\mathrm{BH}}}{m}\right)^{1 / 3} .
$$

In the last expression $M_{\mathrm{BH}}$ is the mass of one of the MBHs, $R_{\star}$ the radius of the star and $m$ its mass. In order to estimate the radius of a star given its mass, we adopt the simple relation $R_{\star} \propto m^{0.6}$ (Demircan \& Kahraman 1991; Gorda \& Svechnikov 1998) with the normalization that a solar mass star has solar radius. Using this in Eq. 3, we can compute the tidal radius in solar radii:

$$
R_{\mathrm{T}, \odot}=1.29 m_{\odot}^{0.6}\left(\frac{M_{\mathrm{BH}}}{m}\right)^{1 / 3},
$$

where $m_{\odot}$ is the mass of the star in solar masses and the prefactor comes from an empirical fit for high-mass stars.

In figure 7 we show the accumulated stellar mass fraction in tidal disruptions for all simulations. Based on this figure and for a time interval of $0.1 \mathrm{Myr}$ after the initialization of the simulations, we can convert the values in tidal disruption events, as shown in table 2. Notice that the rate is actually much higher at the beginning of the simulations, but that result is likely to be affected by our initialisation choices.

\section{DISCUSSION}

In this work we have presented the first realisations of fragmenting discs around a binary of two MBHs in SPH with star formation followed by direct-summation $N$-body simulations of the resulting systems. We have evaluated different fragmentation scenarios based on an approximation for the cooling rate of the gaseous discs and different prescriptions for the growth of protostars. 

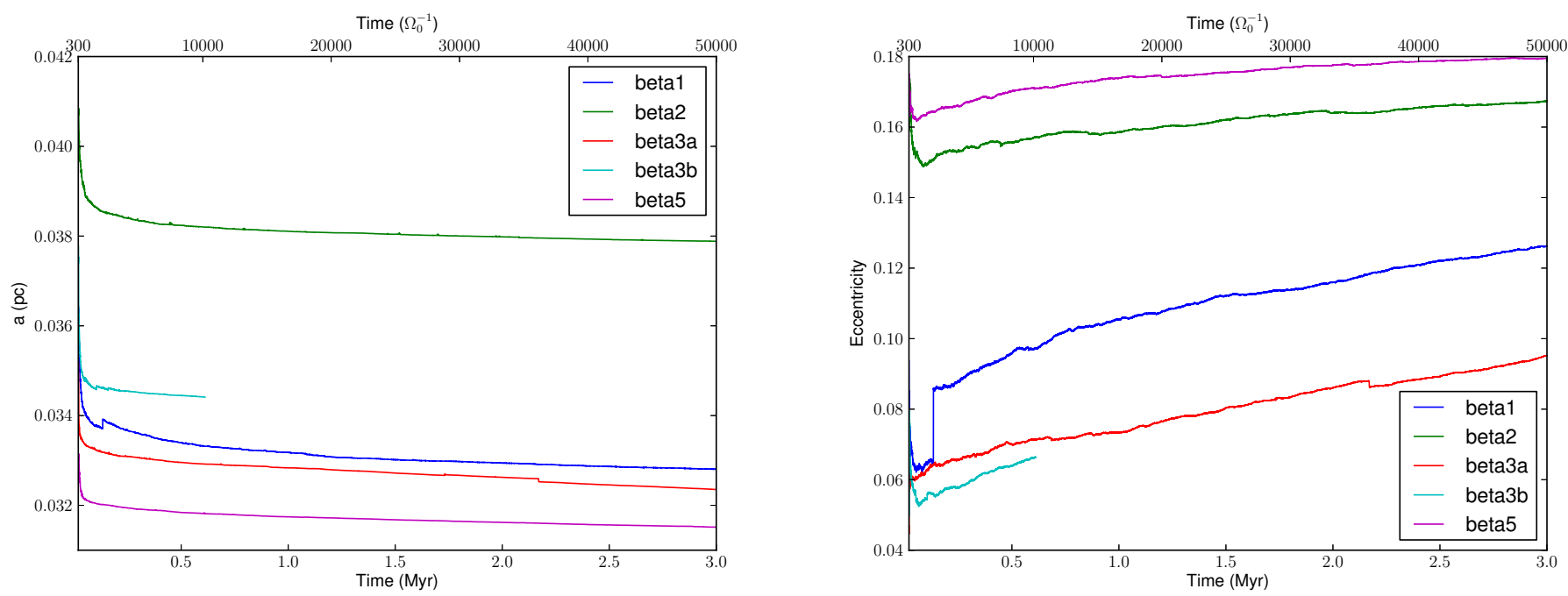

FIG. 4.- Left panel: Evolution of the binary semi-major axis for all cases in the $N$-body simulations in units of the initial orbital period $\Omega_{0}^{-1}$ and in Myrs. Case beta3b was not integrated for more than 10,000 orbits because of numerical issues due to the high number of stars in that simulation. Right panel: Same for the eccentricity.
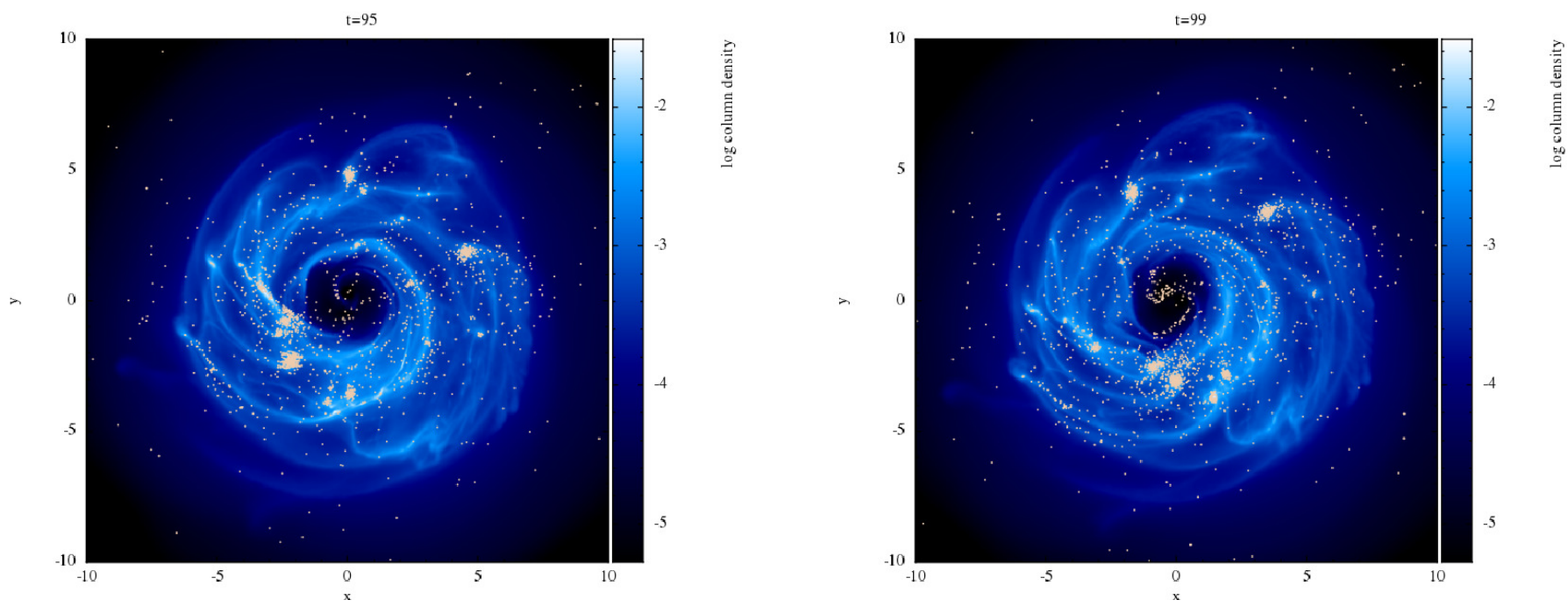

FIG. 5.- Same as figure 1 for the simulation beta 3b. At the SPH time 95 the closest stellar cluster hurls itself on to the binary and leads to an enhancement in the TDEs. We take the position of stars and gas particles from the left panel to start a dedicated direct-summation $N$-body integration which we name after this instant of time, beta3b95.

When the gas is almost completely depleted, we take the masses, positions and velocities of the newly formed stars and feed them to the direct-summation $N$-body integrations with the proviso that if the protostar has a mass above $120 M_{\odot}$, we convert it into an agglomeration of stars following a Plummer profile of radius the Roche radius of the protostar to avoid artificially-created very massive stars.

We find that the rate of decay in our direct $N$-body simulations is slower than the $\approx 10^{-6} \mathrm{yr}^{-1}$ found in the SPH simulations of Cuadra et al. (2009), when scaling to the same masses and separations.

We simulate with a dedicated direct-summation integration the particular case of a simulation in which a cluster of stars that forms during the SPH simulation falls on to the binary, the case beta3b95. For this, we modify NBODY6 to include "gas cloud" particles that allow the infalling cluster to hold together in the dynamical simulation in which we cannot realistically simulate the gas.

Infalling clusters such as this and the scattering of isolated stars lead to a significant number of TDEs. To make an accu- rate estimation, we made a second modification of NBODY6 to implement stellar tidal disruptions, and we find that the event rates lie between $2 \cdot 10^{-5}-\sim 10^{-4}$ per system per year, which lies on the high side of current (uncertain) estimates for the TDE rate in standard galaxies, which typically lie between $10^{-5}-10^{-6} \mathrm{yr}^{-1}$ (Phinney 1989, Magorrian \& Tremaine 1999, Syer \& Ulmer 1999), and lie well within the observed rates (Donley et al. 2002; van Velzen \& Farrar 2012). A particular interesting signature of these TDEs is the "reverberation mapping" response of the circumbinary disc to a burst of emission produced by the TDEs. The light from the burst excites the gas in the disc, producing emission lines. The time-variability of the spectra, the echo of the TDE, during the months after the burst could in principle allow us to constrain the disc structure (Brem, Amaro-Seoane, Cuadra \& Komossa; part II of this paper to be submitted).

While our simulations cannot follow the evolution of the binary for much longer times, it is interesting to ask the question whether the semi-major axis of the binary reaches distances that would lead it to coalesce within a Hubble time 
Initial positions of stars and gas clouds

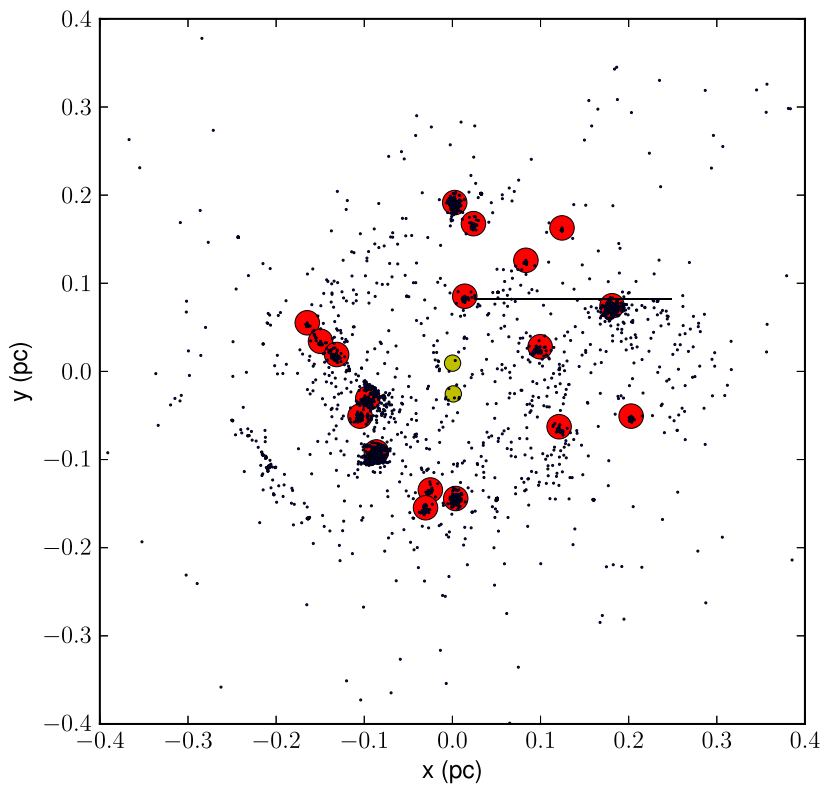

Positions of stars and gas clouds after $\mathrm{T}=1300 \mathrm{yr}$

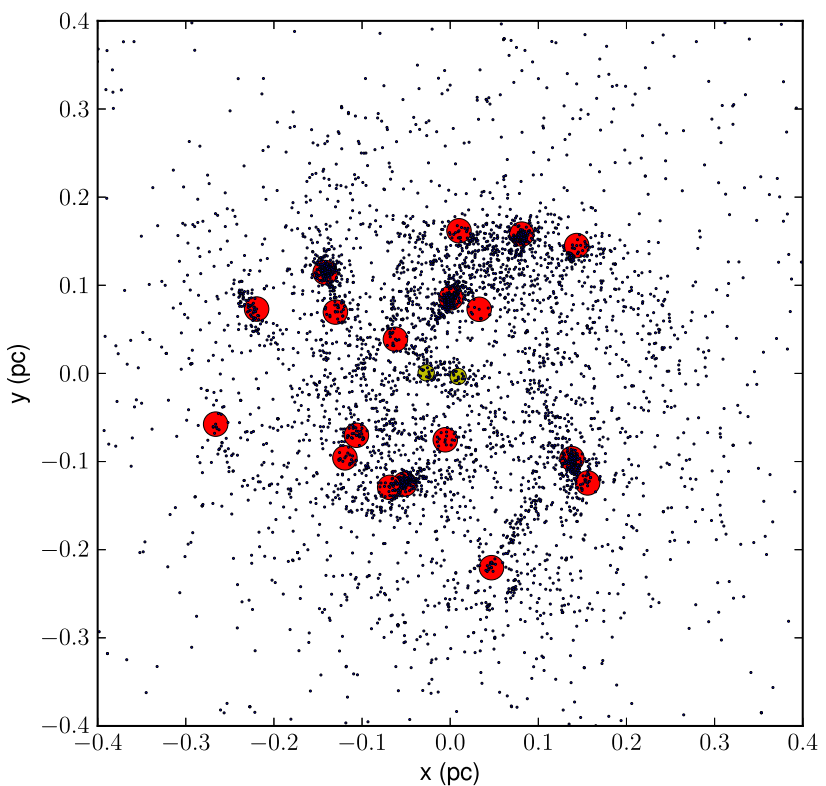

FIG. 6. - Left panel: Initial configuration in the x-y plane perpendicular to the angular momentum vector of the system for simulation beta $3 \mathrm{~b} 95$ of table 1 . Stars are shown with black dots, gas clouds with red circles. The MBHs are depicted with green circles. Right panel: The same system after the cluster falls on to the binary, after $\sim 1,300$ yrs. Note the enhanced number of stars in the vicinity of the binary. This translates in a larger number of TDEs.

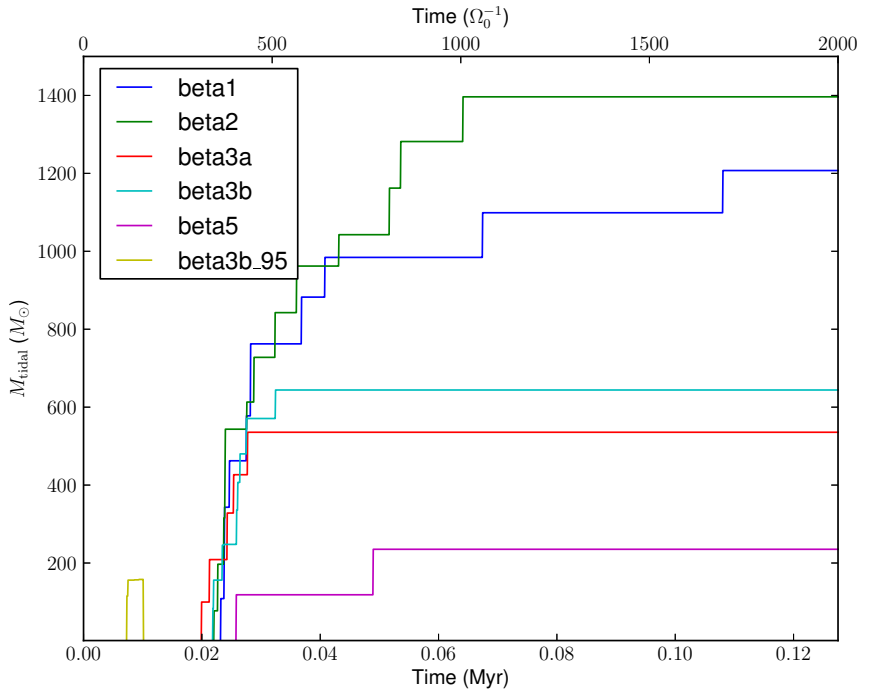

FIG. 7.- Accumulated stellar mass in tidal disruptions for the different simulations of table 1 Since we only track them in the $N$-body simulations, the curves start at $T=300 \Omega_{0}^{-1}$ but for model bet a 3b95, which as explained before, had a dedicated run. Because we cannot simulate realistically more than one infall of the cluster, we stop it after the first periapsis passage.

because of the emission of gravitational radiation, measurable in a LISA-like detector such as eLISA (Amaro-Seoane et al.2012a). For this, the binary has to shrink from an initial semi-major of $a \approx 0.04 \mathrm{pc}$ down to $a \approx 0.003 \mathrm{pc}$. This corresponds to an increase of orbital binding energy of about one order of magnitude. The net change in binding energy after an interaction with one bound star of mass $m_{\star}$ can be estimated as $\Delta E_{\star}=G m_{\star} M_{\mathrm{bbh}} / a$. We start the direct-summation simulations with a ratio of stellar mass to $\mathrm{MBH}$ binary mass of $\approx 10 \%$, so that ab definitio the stellar mass that is formed is not enough for the binary to shrink down to the phase in which the evolution is dominated by gravitational radiation. Indeed, if we consider all stars in the disc to be ejected, we estimate in the limit of this low mass ratio that the total ef- fect of the stellar disc is of about $\delta E_{\mathrm{tot}}=G q M_{\mathrm{bbh}}^{2} / a$, where $q$ is the mass ratio of stellar mass to $\mathrm{BH}$ mass. Following an argument similar to e.g. Quinlan (1996); Sesana et al. (2007), if we compare this to the orbital energy at semi-major axis $a$, one finds that the relative change after ejecting all the stars is $\delta E_{\text {tot }} / E \approx q / \nu$, where $\nu=0.2$ is the symmetric mass ratio, well below what would be necessary to shrink the binary by one order of magnitude. We note that indeed ejecting half of the stellar mass only shrinks the binary semi-major axis by $<25 \%$, as we see in the first 3 Myr of our $N$-body simulations, in figure 8 . While this is true for our specific scenario, we note that further episodes of gas inflow towards the centre could potentially trigger more episodes of star formation in the disc, which would lead to star scattering and a further skrinkage. Moreover, while we have focused on the effect of stars formed in-situ on the binary, but the system will be surrounded by a stellar cusp that constitutes an additional source of shrinkage for the binary. The supply of stars that will interact with it can be enhanced by additional mechanisms in a more realistic picture than that of an isolated, spherically symmetric galactic nucleus, as we discussed in the introduction.

PAS is indebted to the Universidad Católica for their hospitality and support for a two-week visit, as well as to Jura Borissova and her group from Universidad Valparaíso for a short visit, and to Cristián Maureira, for his help and discussions on the way to Valparaíso. JC acknowledges support from FONDAP (15010003), FONDECYT (11100240), Basal (PFB0609), VRI-PUC (Inicio 16/2010) and the European Commission's Framework Programme 7 through the Marie-Curie IRSES project LACEGAL (PIRSES-GA-2010269264). This work was supported in part by the National Science Foundation under Grant No. 1066293 and the hospitality of the Aspen Center for Physics and by the Transregio 7 "Gravitational Wave Astronomy" financed by the Deutsche Forschungsgemeinschaft DFG (German Research 


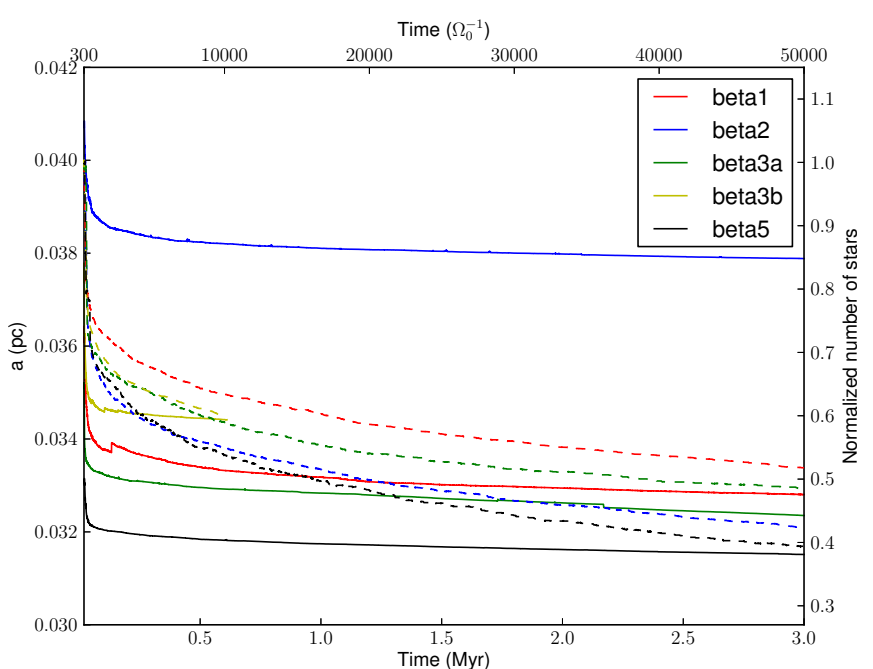

FIG. 8.- Same as the left panel of figure 4 but including the number of stars in each simulation, for the same colour but in dashed lines. By the end of our numerical treatment we have lost at least $50 \%$ in all cases.
Foundation).

\section{REFERENCES}

Aarseth, S. J. 1999, The Publications of the Astronomical Society of the Pacific, 111

- 2003, Gravitational N-Body Simulations (ISBN 0521432723. Cambridge, UK: Cambridge University Press, November 2003.)

Alexander, R. D., Armitage, P. J., \& Cuadra, J. 2008, MNRAS, 389, 1655

Amaro-Seoane, P. 2012, ArXiv e-prints

Amaro-Seoane, P. et al. 2012a, Accepted for publication at GW Notes

- 2012b, Classical and Quantum Gravity, 29, 124016

Amaro-Seoane, P., Eichhorn, C., Porter, E. K., \& Spurzem, R. 2010, MNRAS, 401, 2268

Amaro-Seoane, P., \& Freitag, M. 2006, ApJ Lett., 653, L53

Amaro-Seoane, P., Miller, M. C., \& Freitag, M. 2009, ApJ Lett., 692, L50

Begelman, M. C., Blandford, R. D., \& Rees, M. J. 1980, Nat, 287

Berczik, P., Merritt, D., Spurzem, R., \& Bischof, H.-P. 2006, ApJ Lett., 642, L21

Cuadra, J., Armitage, P. J., Alexander, R. D., \& Begelman, M. C. 2009, MNRAS, 393, 1423

Cuadra, J., Nayakshin, S., Springel, V., \& Di Matteo, T. 2006, MNRAS, 366,358

Demircan, O., \& Kahraman, G. 1991, Astrophysics and Space Science, 181

Donley, J. L., Brandt, W. N., Eracleous, M., \& Boller, T. 2002, 124, 1308, arXiv:astro-ph/0206291

Dotti, M., Colpi, M., Haardt, F., \& Mayer, L. 2007, MNRAS, 379, 956

Escala, A., Larson, R. B., Coppi, P. S., \& Mardones, D. 2004, ApJ, 607, 765 - 2005, ApJ, 630, 152

Gammie, C. F. 2001, ApJ, 553, 174

Gorda, S. Y., \& Svechnikov, M. A. 1998, Astronomy Reports, 42

Hopman, C., \& Alexander, T. 2006, ApJ, 645, 1152

Khan, F. M., Berentzen, I., Berczik, P., Just, A., Mayer, L., Nitadori, K., \& Callegari, S. 2012, ApJ, 756, 30

Khan, F. M., Just, A., \& Merritt, D. 2011, ApJ, 732, 89

Kustaanheimo, P. E., \& Stiefel, E. L. 1965, J. Reine Angew. Math.

Levin, Y. 2007, MNRAS, 374, 515
Lodato, G. 2007, Nuovo Cimento Rivista Serie, 30, 293

Magorrian, J., \& Tremaine, S. 1999, MNRAS, 309

Mayer, L., Kazantzidis, S., Madau, P., Colpi, M., Quinn, T., \& Wadsley, J. 2007, Science, 316, 1874

McMillan, S. L. W., \& Portegies Zwart, S. F. 2003, ApJ, 596, 314

Merritt, D., \& Milosavljević, M. 2005, Living Reviews in Relativity, 8, 8

Merritt, D., \& Vasiliev, E. 2010, ArXiv e-prints

Milosavljević, M., \& Merritt, D. 2003, ApJ, 596

Nayakshin, S., Cuadra, J., \& Springel, V. 2007, MNRAS, 379, 21

Paardekooper, S.-J. 2012, MNRAS, 421, 3286

Perets, H. B., \& Alexander, T. 2008, ApJ, 677, 146

Phinney, E. S. 1989, in IAU Symposium, Vol. 136, The Center of the Galaxy, ed. M. Morris, 543

Plummer, H. C. 1911, MNRAS, 71

Portegies Zwart, S. F., Baumgardt, H., McMillan, S. L. W., Makino, J., Hut, P., \& Ebisuzaki, T. 2006, ApJ, 641, 319

Preto, M., Berentzen, I., Berczik, P., \& Spurzem, R. 2011, ApJ Lett., 732, L26

Price, D. J. 2007, Publications of the Astronomical Society of Australia, 24, 159

Quinlan, G. D. 1996, New Astronomy, 1

Rice, W. K. M., Lodato, G., \& Armitage, P. J. 2005, MNRAS, 364, L56 Roedig, C., Dotti, M., Sesana, A., Cuadra, J., \& Colpi, M. 2011, MNRAS, 415, 3033

Roedig, C., Sesana, A., Dotti, M., Cuadra, J., Amaro-Seoane, P., \& Haardt, F. 2012, A\&A, 545, A127

Sesana, A., Haardt, F., \& Madau, P. 2007, ArXiv e-prints, 710

Springel, V. 2005, MNRAS, 364, 1105

Springel, V., Di Matteo, T., \& Hernquist, L. 2005, MNRAS, 361, 776

Springel, V., Yoshida, N., \& White, S. D. M. 2001, New Astronomy, 6, 79

Syer, D., \& Ulmer, A. 1999, MNRAS, 306

van Velzen, S., \& Farrar, G. R. 2012, ArXiv e-prints 